\title{
CURRENT PERSPECTIVES REGARDING THE APPLICATION AND INCORPORATION OF SILVER NANOPARTICLES INTO DENTAL BIOMATERIALS
}

\author{
MIHAI FLAVIU ŞUHANI ${ }^{1}$, GRIGORE BĂCIUȚ ${ }^{1}$, MIHAELA BĂCIUȚ ${ }^{2}$, \\ RALUCA ŞUHANI $^{3}$, SIMION BRAN ${ }^{1}$
}

\author{
${ }^{1}$ Department of Oral and Maxillofacial Surgery, Iuliu Hatieganu University of \\ Medicine and Pharmacy, Cluj-Napoca, Romania \\ ${ }^{2}$ Department of Implantology and Maxillofacial Surgery, Iuliu Hatieganu \\ University of Medicine and Pharmacy, Cluj-Napoca, Romania \\ ${ }^{3}$ Department of Pediatric Dentistry, Iuliu Hatieganu University of Medicine and \\ Pharmacy, Cluj-Napoca, Romania
}

\begin{abstract}
Introduction. The key idea of nanotechnology is to construct and preserve functional structures by means of exploiting atoms and molecules. Nanotechnology has proven to be crucial in pharmacological medicine, tissue engineering, clinical diagnosis, long term conservation of biological tissues in a cryogenic state, protein detection, tumor destruction and magnetic resonance imaging.

The aim of this paper is to review the literature on the specific characteristics of nanostructured materials, their applications and advantages that they bring to dentistry.

Method. We conducted an electronic scientific database research that included PubMed, Cochrane and Medline. The following keywords were used: nanotechnology, nanodentistry and silver nanoparticles. Initially 1650 original articles were retrieved from the these mentioned international databases, which were screened in detail. We included literature reviews that dealt with the comprehensive applications of nanostructured particles and silver nanoparticles in particular, in all fields of contemporary dentistry. Case reports, clinical trials, editorials and opinion letters were excluded in the first phase of our research. Fifty two articles met all the selection criteria and were ultimately selected and reviewed.

Results. Nanotechnology deals with the production of various types of nanomaterials with potential applications in the field of biomedicine. Silver nanoparticles have the capacity to eliminate dental caries producing bacteria or repair teeth enamel with signs of dental decay. Nanodentistry will allow better oral health by use of nanostructured materials. Treatment opportunities that nanotechnology has to offer in contemporary dentistry include local anesthesia, permanent treatment of dental hypersensitivity, orthodontic and oral health care with nanorobotic dentifrice.

Conclusion. The studies that we reviewed are largely in favor of nanotechnology and nanostructured materials, highlighting their qualities and enhancements they bring to the field of dentistry. Although many of these products that benefit from silver nanoparticles properties are still expensive and exclusive, we can foresee major improvements and demand regarding dental biomaterials with nanoparticles incorporated in the near future.
\end{abstract}

Keywords: nanotechnology, nanodentistry, silver nanoparticles

Manuscript received: 25.01 .2018

Received in revised form: 05.03.2018

Accepted: 17.03.2018

Address for correspondence: gbaciut@umfcluj.ro 


\section{Introduction}

The antibacterial properties of the silver nanoparticles (AgNPs) have been utilized with success in various medical domains. AgNPs have been used in several fields of dentistry, as endodontics [1,2], prostheses [3], implantology [4,5], orthodontics and restorative dentistry [6-8]. AgNPs incorporation goal is to eliminate microbial colonization over dental biomaterials, thus improving the overall oral health status.

\section{Benefits of AgNPs}

AgNPs are essentially clusters of silver atoms that have dimensions from 1 to $100 \mathrm{~nm}$. Nanoparticles come in different forms and structures like nanospheres, nanopores, nanocapsules, dendrimers, quantum dots, nanoshells or nanotubes.

Nano-silver, comprising AgNPs, presents antibacterial, antiviral as well as anti-inflammatory effects.

The antimicrobial qualities of silver are directly correlated with the amount and discharge rate. Metallic silver is inert, but when it overreacts with moisture or fluids, ionization results. The ionized silver is extremely reactive, as it attaches to tissue proteins leading to structural modifications in the bacterial cell wall, in the nuclear membrane, conducting cell distortion and death [9]. The influence of silver ions on bacteria can be noticed by the structural and morphological changes they generate. Silver ions come in contact with the three main elements of a bacterial cell in order to produce a germicidal effect: the peptidoglycan cell wall $[10,11]$, plasma membrane [12] and bacterial DNA [13]. Silver ions also interact with enzymes involved in primal processes at the cellular level. It is described that silver ions also cause the lysis of bacterial cells [14].

Nano-silver possesses extraordinary biological activities, such as antiviral properties $[15,16]$. AgNPs are effective and powerful virucides as they inactivate HIV molecules in a rapid manner, exercising their activity at an early phase of viral replication and at post-entry stages [17]. The effects of nano-silver depends on the fusion inhibition of the virus into the host cell, since blocking HIV entry into its target cells can lead to the suppression of viral infectivity, replication and cytotoxicity induced by viruscell interaction [18].

\section{Material and methods}

This review offers an insight on the latest investigations regarding the incorporation of silver nanoparticles in modern dental biomaterials. An extensive review on this matter may prove to be excessive, therefore we selected quintessential examples to be reported in this research.

An electronic scientific database research was conducted on "open access" journals. The search databases used included the following: PubMed, Cochrane and Medline. We adjusted the search filters for the last 10 years, from 2007 onwards. Various combinations of the following keywords: nanotechnology, nanodentistry and silver nanoparticles were utilised. One thousand six hundred and fifty original articles were retrieved from these international databases. All the published articles identified were subjected to a thorough screening based on title and abstract. We also inspected the reference lists of each of these articles in order to detect studies that were previously omitted.

The inclusion and exclusion criteria implemented are listed below:

\section{Inclusion criteria:}

1. original articles and literature reviews that considered the comprehensive applications of nanostructured particles and silver nanoparticles in particular, in all fields of contemporary dentistry;

2. year of publication (2007-2017) using the topics (nanotechnology, nanodentistry and silver nanoparticles);

3. studies written in English.

\section{Exclusion criteria:}

1. abstracts without free full text articles, editorials, case reports, opinion letters, clinical trials;

2. poorly designed articles;

3. studies that were published prior to 2007 ;

4. full text articles written in another language than English.

\section{Results and discussion}

Using the search strategy presented above, between 2007-2017, a number of 1650 articles were found and screened. One hundred and sixty studies (160) were published in other languages than English, 145 studies were poorly designed articles and 1293 were abstracts without full text, case reports, clinical trials, editorial or opinion letters. Fifty two articles met all the selection criteria and were ultimately selected.

\section{Nanotechnology in contemporary dentistry Periodontal disease}

A wide array of drug delivery systems have been investigated for periodontitis treatment, including dental fibers, gels, films, strips and compacts [19]. Research is centered around nanodelivery systems for an efficient and targeted delivery of drugs to the periodontal pocket. Nanodelivery systems explored include nanocomposite hydrogels [20], nanoparticles, nanoemulsions [21] .

Trichlosan, minocycline, Harungana madagascariensis extract, tetracycline, chlorhexidine and other synthetic or herbal drugs have been incorporated into nanodelivery systems for treating periodontitis. Polymers like chitosan and polypropylene are also being investigated as matrices for drug delivery inside the periodontal pocket.

\section{Oral and maxillofacial surgery}

The key factor for a successful dental implant is osteoblast proliferation. This goal has been realized by creating nano-sized particles on the surface of the implant. Nanotopography of the implant surface influences 
osteogenic cells while the surface morphology enhances osteoblast adhesion. The morphology of the surface increases the functional area thus providing a greater implant surface area that can interact with the biologic environment around it $[22,23]$.

Nanotechnology can emulate natural bone structure, which is comprised of organic compounds reinforced with inorganic materials. Nanocrystals present a loose structure, with nanopores in between the crystals. The surfaces of these pores are engineered with contribution from silica in order to adsorbe protein. Maxillofacial traumatic bone defects can be treated using hydroxyapatite nanoparticles [24].

\section{Dentinal hypersensitivity}

Dentinal hypersensitivity is caused by pressure changes transmitted hydrodynamically to the dental pulp. The dentinal tubules of a hypersensitive tooth have twice the diameter and eight times the surface density of those in non sensitive teeth. Dental nanorobots could selectively and precisely close damaged tubules thus offering a quick and permanent cure [24].

\section{Glass ionomers}

Glass ionomers with $\mathrm{TiO}_{2}$ nanoparticles incorporated present greater resistance to fracture, flexural and compressive strength compared to the unmodified conventional glass ionomers. The addition of these nanoparticles to the standard glass ionomer did not affect dentine bond strength or fluoride release. Glass ionomer including $\mathrm{TiO}_{2}$ nanoparticles presented the most potent antibacterial activity (against Streptococcus Mutans) compared to unmodified glass ionomers [25].

\section{Restorative composites}

Nanotechnology has allowed the production of nanofiller particles [26] which are added individually or as clusters into composite resins. Silver nanoparticles offer composites a smoother surface and superior esthetic properties. Composite resins with nanoparticles are easier to handle and have greater strength and abrasion resistance. Resin containing nanostructured particles are used in a wider array than composites with hybrid and microfilled fillers [27].

Recently, quaternary ammonium polyethylene imine nanoparticles were developed for extra antibacterial properties of restorative composite resins. In recent studies it was described how these nanoparticles completely suppressed the growth of Streptococcus Mutans bacteria, with an antibacterial activity that lasted for up to 3 months $[25,28]$.

It has been observed that no relevant difference in terms of enamel lesions and cracks occurred after debracketing of orthodontic brackets bonded with flowable orthodontic composite compared with traditional orthodontic composite [29].

\section{Orthodontics}

Katz et al. [30] reported a reduction in the frictional force produced by orthodontic movement by coating
NiTi and stainless steel wires with tungsten disulfide nanoparticles (IF-WS2). Cao et al. [31] reported in their research that orthodontic brackets coated with a nitrogendoped titanium oxide film showed increased antimicrobial features, effective in prevention of dental enamel demineralization, gingivitis and periodontal disease.

Orthodontic nanorobots have the potential to manipulate periodontal tissues, allowing rapid and painless tooth alignment, horizontal and vertical repositioning as well as rapid and effective tissue repair [24,30].

\section{Dental prophylaxis}

Dental robots incorporated in mouthwash or toothpaste can clean supragingival and subgingival surfaces, metabolizing trapped organic matter into odorless vapors while also performing continuous calculus and plaque debridement. These nanorobots are securely inactivated when swallowed [24]. Use of a toothpaste incorporating nanosized calcium carbonate empowered remineralization of early enamel lesions [32].

\section{Imagery}

Advances in imaging techniques used in modern day dentistry and oral surgery are anticipated with the support of nanotechnology. In digital radiographs acquired by nanophosphor scintillators, the radiation dosage is massively diminished and high-quality images are obtained on a regular basis [33].

\section{AgNPs in dentistry}

Materials based on silver nanoparticles

Means of incorporation

AgNPs utilized in dental biomaterials are incorporated through different techniques. The most common method for composite resin and adhesive systems is adding a monomer, usually 2-tert-butylaminoethyl methacrylate, in order to improve Ag salt solubility in the resin solution [34-36].

For dental implants, the process is distinct. Titanium samples are soaked in $\mathrm{AgNO} 3$ solutions, rinsed with deionized water, dried and irradiated with ultraviolet light from a high-pressure Hg lamp. This process enables the manufacture of samples with different $\mathrm{Ag}$ concentrations, depending on the concentration of $\mathrm{AgNO}_{3}$ solution [37].

\section{Composite resins}

AgNPs were first introduced into dental composites in order to induce antimicrobial properties and enhance biocompatibility $[14,38,39]$. The altered tissue conditioner compound with silver nanoparticles showed antimicrobial properties against bacteria such as Streptococcus mutans, Staphylococcus aureus and Candida albicans incorporated after a 24-h or 72-h incubation [40].

By adding silver hydrosol, light cured flowable composites function as an antimicrobial product. The silver hydrosol can be released from the resin composite matrix in order to decrease the incidence of tooth decay [41]. Yoshida et al. [42] demonstrated the antibacterial activity of composite resins containing high concentrations 
of silver nanoparticles (up to 10\%) against Streptococcus mutans [42]. AgNPs exhibit an antimicrobial effect on Streptococcus mutans, the main causative microorganism of caries. The antimicrobial effects of AgNPs-containing restorative composites might reduce the development of recurrent dental caries. They also tend to favor an increase of the longevity of tooth restorations and are effective in reducing bacterial biofilm on teeth and restorations, without modifying the mechanical properties or cytotoxicity of resins and adhesives.

\section{Implants}

In order to obstruct biofilm formation over implants' surface, antimicrobial coatings have been developed. Unfortunately, most of them present subpar long-term antibacterial effect with the possibility of generating resistant strains [43-45]. In this context of use, AgNPs integration to implant surface has been suggested [46,47], as it could produce coatings with long-term antimicrobial properties by controlling Ag release [5]. Zhao et al. [5] incorporated AgNPs into titania nanotubes on titanium (Ti) implants, using silver nitrate immersion and UV light radiation. Assessing the antibacterial effect against Staphylococcus aureus, the results evidenced inhibition of planktonic pathogens during the first days. AgNPs-coated Ti implants presented the capability to prevent bacteria adhesion for up to 30 days, which is considered favorable in order to prevent post-implant therapy infection at early stages [46].

\section{Impression materials}

Alginate impression powders can be combined with water that contains AgNPs in order to create an impression material that exhibits antimicrobial activity. This will significantly reduce cross contamination by bacteria, viruses or fungi from the impression to the cast model [48].

\section{Endodontics}

Antimicrobial root canal sealers/cements with silver hydrosol added in their composition, are useful in the permanent obturation of root canal(s) following removal of the infected dental pulp and placement of medicaments/ materials inside the root canal(s).

Iranian researchers have introduced nanosilver incorporated in gutta-percha, as a mean to improve its antibacterial effect [49]. This new material, which is standard gutta-percha coated with AgNPs, has shown significant effect against Staphylococcus aureus, Candida albicans and Escherichia coli. Shantiaee et al. [50] have tested the biocompatibility of this new material, by comparing the cytotoxicity of nanosilver-coated guttapercha and normal gutta-percha on mouse fibroblasts. After 24 hours, nanosilver gutta-percha presented cytotoxicity values similar to normal gutta-percha. After one week, it reached the lowest level among the tested materials [50].

\section{Acrylic resins}

In a study performed by Monteiro et al. [51] AgNPs were incorporated in a commercial acrylic resin, in different concentrations $(0.05 \%, 0.5 \%$ and $5 \%$ of AgNPs, by mass). The mechanical properties of the modified resin were evaluated, as well as of the unmodified resin. The flexural strength test was performed. It was observed that all groups presented similar flexural resistance, suggesting that AgNPs incorporation does not affect the mechanical properties of acrylic resins [51].

\section{Cements}

Dental cements like glass ionomers or epoxy resin cements can be impregnated with silver hydrosol. A significant number of permanent dental cements can be mixed or combined with silver hydrosol. By adding the silver hydrosol to these cements, one is able to provide a continuous dynamic antimicrobial bacteriostatic environment capable of reducing bacterial bioburden and thus postoperative inflammation, infection and sensitivity, which are particularly important with vital teeth [52].

\section{Conclusion}

AgNPs have risen as one of the most potent and useful antibacterial agents due to their large surface area to volume ratios. AgNPs can be used as effective growth inhibitors of various microorganisms. Nanomaterials can be manipulated in order to accomplish better efficiency and to facilitate their applications. Oral health care services will become easier for dental surgeons and dentists alike, more acceptable to patients with a more favorable outcome.

Optimal utilization of the advantages and opportunities offered by nanotechnology and AgNPs in particular in day-to-day clinical dental practice will facilitate improvements in oral health. Nanotechnology carries potential for misuse if not properly controlled. The risk and toxicity of these nanostructured materials need extensive research, as well as cost effectiveness and patient acceptance. The long-term antibacterial and clinical effects of AgNPs on medical and dental biomaterials should be analyzed in future research.

\section{References}

1. Samiei M, Aghazadeh M, Lotfi M, Shakoei S, Aghazadeh Z, Vahid Pakdel SM. Antimicrobial efficacy of mineral trioxide aggregate with and without silver nanoparticles. Iran Endod J. 2013;8(4):166-170.

2. Lotfi M, Vosoughhosseini S, Ranjkesh B, Khani S, Saghiri M, Zand V. Antimicrobial efficacy of nanosilver, sodium hypochlorite and chlorhexidine gluconate against Enterococcus faecalis. African Journal of Biotechnology. 2011;10(35):6799-6803.

3. Nam KY. In vitro antimicrobial effect of the tissue conditioner containing silver nanoparticles. J Adv Prosthodont. 2011;3(1):2024.

4. Flores CY, Diaz C, Rubert A, Benítez GA, Moreno MS, Fernández Lorenzo de Mele MA, et al. Spontaneous adsorption of silver nanoparticles on $\mathrm{Ti} / \mathrm{TiO} 2$ surfaces. Antibacterial effect on Pseudomonas aeruginosa. J Colloid Interface Sci. 2010;350(2):402-408.

5. Zhao L, Wang H, Huo K, Cui L, Zhang W, Ni H, et al. Antibacterial nano-structured titania coating incorporated with 
silver nanoparticles. Biomaterials. 2011;32(24):5706-5716. 6. Durner J, Stojanovic M, Urcan E, Hickel R, Reichl FX. Influence of silver nano-particles on monomer elution from lightcured composites. Dent Mater. 2011;27(7):631-636.

7. Cheng L, Weir MD, Xu HH, Antonucci JM, Kraigsley AM, Lin NJ, et al. Antibacterial amorphous calcium phosphate nanocomposites with a quaternary ammonium dimethacrylate and silver nanoparticles. Dent Mater. 2012;28(5):561-572.

8. Cheng L, Weir MD, Xu HH, Antonucci JM, Lin NJ, LinGibson S, et al. Effect of amorphous calcium phosphate and silver nanocomposites on dental plaque microcosm biofilms. J Biomed Mater Res B Appl Biomater. 2012;100(5):1378-1386.

9. Castellano JJ, Shafii SM, Ko F, Donate G, Wright TE, Mannari RJ, et al. Comparative evaluation of silver-containing antimicrobial dressings and drugs. Int Wound J. 2007;4:114-122. 10. Yamanaka M, Hara K, Kudo J. Bactericidal actions of a silver ion solution on Escherichia coli, studied by energy-filtering transmission electron microscopy and proteomic analysis. Appl Environ Microbiol. 2005;71:7589-7593.

11. Raffi M, Hussain F, Bhatti TM, Akhter JI, Hameed A, Hasan MM. Antibacterial Characterization of Silver Nanoparticles against E. Coli ATCC-15224. J Mater Sci Technol. 2008;24:192196.

12. Jung WK, Koo HC, Kim KW, Shin S, Kim SH, Park YH. Antibacterial activity and mechanism of action of the silver ion in Staphylococcus aureus and Escherichia coli. Appl Environ Microbiol. 2008;74:2171-2178.

13. Shrivastava S, Bera T, Roy A, Singh G, Ramachandrara P, Dash D. Characterization of enhanced antibacterial effects of novel silver nanoparticles. Nanotechnology. 2007;18:225103. doi: 10.1088/0957-4484/18/22/225103.

14. Morones JR, Elechiguerra JL, Camacho A, Holt K, Kouri JB, Ramírez JT, et al. The bactericidal effect of silver nanoparticles. Nanotechnology. 2005;16:2346-2353.

15. Rai M, Yadav A, Gade A. Silver nanoparticles as a new generation of antimicrobials. Biotechnol Adv. 2009;27:76-83.

16. Duran N, Marcarto PD, De Souza GIH, Alves OL, Esposito E. Antibacterial effect of silver nanoparticles produced by fungal process on textile fabrics and their effluent treatment. Journal of Biomedical Nanotechnology. 2007;3:203-208.

17. Al-Jabri AA, Alenzi FQ. Vaccines, virucides and drugs against HIV/AIDS: hopes and optimisms for the Future. Open AIDS J. 2009;3:1-3.

18. Lara HH, Ayala-Nuñez NV, Ixtepan-Turrent L, RodriguezPadilla C. Mode of antiviral action of silver nanoparticles against HIV-1. J Nanobiotechnology. 2010 Jan 20;8:1. doi: 10.1186/14773155-8-1.

19. Taresh AD, Juma SS. Local drug delivery systems for treating periodontal diseases (A review of literature.) Journal Baghdad College of Dentistry.2013;25:79-85.

20. Jayakaran TG, Arjunkumar R. Nanocomposite hydrogels as local drug delivery in periodontics. Journal of Pharmaceutical Sciences Research. 2013;5:277-278.

21. Yao W, Xu P, Pang Z, Zhao J, Chai Z, Li X, et al. Local delivery of minocycline-loaded PEG-PLA nanoparticles for the enhanced treatment of periodontitis in dogs. Int J Nanomedicine. 2014;9:3963-3970.

22. Meirelles L, Currie F, Jacobsson M, Albrektsson T, Wennerberg A. The effect of chemical and nanotopographical modifications on the early stages of osseointegration. Int J Oral Maxillofac Implants. 2008;23:641-647.
23. Park JW, Kim HK, Kim YJ, An CH, Hanawa T. Enhanced osteoconductivity of micro-structured titanium implants (XiVE $\mathrm{S}$ CELLplus) by addition of surface calcium chemistry: a histomorphometric study in the rabbit femur. Clin Oral Implants Res. 2009;20:684-690.

24. Kumar SR, Vijayalakshmi R. Nanotechnology in dentistry. Indian J Dent Res. 2006;17:62-65.

25. Elsaka SE, Hamouda IM, Swain MV. Titanium dioxide nanoparticles addition to a conventional glass-ionomer restorative: influence on physical and antibacterial properties. J Dent. 2011;39:589-598.

26. Jung M, Sehr K, Klimek J. Surface texture of four nanofilled and one hybrid composite after finishing. Oper Dent. 2007;32:4552.

27. Yesil ZD, Alapati S, Johnston W, Seghi RR. Evaluation of the wear resistance of new nanocomposite resin restorative materials. J Prosthet Dent. 2008;99:435-443.

28. Lee HH, Chou KS, Shih ZW. Effect of nano-sized silver particles on the resistivity of polymeric conductive adhesives. International Journal of Adhesion and Adhesives. 2005;25:437441.

29. Tecco S, Tetè S, D'Attilio M, Festa F. Enamel surface after debracketing of orthodontic brackets bonded with flowable orthodontic composite. A comparison with a traditional orthodontic composite resin. Minerva Stomatol. 2008;57:81-94.

30. Redlich M, Katz A, Rapoport L, Wagner HD, Feldman Y, Tenne R. Improved orthodontic stainless steel wires coated with inorganic fullerene-like nanoparticles of WS(2) impregnated in electroless nickel-phosphorous film. Dent Mater. 2008;24:16401646.

31. Cao B, Wang Y, Li N, Liu B, Zhang Y. Preparation of an orthodontic bracket coated with an nitrogen-doped $\mathrm{TiO}(2-\mathrm{x}) \mathrm{N}(\mathrm{y})$ thin film and examination of its antimicrobial performance. Dent Mater J. 2013;32:311-316.

32. Nakashima S, Yoshie M, Sano H, Bahar A. Effect of a test dentifrice containing nano-sized calcium carbonate on remineralization of enamel lesions in vitro. J Oral Sci. 2009;51:69-77.

33. Mupparapu M. New nanophosphor scintillators for solid-state digital dental imagers. Dentomaxillofac Radiol. 2006;35:475476.

34. Cheng YJ, Zeiger DN, Howarter JA, Zhang X, Lin NJ, Antonucci JM, et al. In situ formation of silver nanoparticles in photocrosslinking polymers. J Biomed Mater Res B Appl Biomater. 2011;97(1):124-131.

35. Li F, Weir MD, Chen J, Xu HH. Comparison of quaternary ammonium-containing with nano-silver-containing adhesive in antibacterial properties and cytotoxicity. Dent Mater. 2013;29(4):450-461.

36. Melo MA, Cheng L, Weir MD, Hsia RC, Rodrigues LK, Xu HH. Novel dental adhesive containing antibacterial agents and calcium phosphate nanoparticles. J Biomed Mater Res B Appl Biomater. 2013;101(4):620-629.

37. Zhao L, Chu PK, Zhang Y, Wu Z. Antibacterial coatings on titanium implants. J Biomed Mater Res B Appl Biomater. 2009;91(1):470-480.

38. Kim JS, Kuk E, Yu KN, Kim JH, Park SJ, Lee HJ, et al. Antimicrobial effects of silver nanoparticles. Nanomedicine. 2007;3:95-101.

39. Jandt KD, Al-Jasser AM, Al-Ateeq K, Vowles RW, Allen GC. Mechanical properties and radiopacity of experimental glass- 
silica-metal hybrid composites. Dent Mater. 2002;6:429-435. 40. Herrera M, Carrión P, Baca P, Liébana J, Castillo A. In vitro antibacterial activity of glass-ionomer cements. Microbios. 2001; 104:141-148.

41. Li Z, Lee D, Sheng X, Cohen RE, Rubner MF. Two-level antibacterial coating with both release-killing and contact-killing capabilities. Langmuir. 2006;22:9820-9823.

42. Yoshida K, Tanagawa M, Matsumoto S, Yamada T, Atsuta M. Antibacterial activity of resin composites with silver-containing materials. Eur J Oral Sci. 1999;107:290-296.

43. Hendriks JG, van Horn JR, van der Mei HC, Busscher HJ. Backgrounds of antibiotic-loaded bone cement and prosthesisrelated infection. Biomaterials. 2004;25(3):545-556.

44. Campoccia D, Montanaro L, Arciola CR. The significance of infection related to orthopedic devices and issues of antibiotic resistance. Biomaterials. 2006;27(11):2331-2339.

45. Arciola CR, Baldassarri L, Campoccia D, Creti R, Pirini V, Huebner J, et al. Strong biofilm production, antibiotic multiresistance and high gelE expression in epidemic clones of Enterococcus faecalis from orthopaedic implant infections. Biomaterials. 2008;29(5):580-586.

46. Hardes J, Ahrens H, Gebert C, Streitbuerger A, Buerger H, Erren M, et al. Lack of toxicological side-effects in silver-coated megaprostheses in humans. Biomaterials. 2007;28(18):2869-2875. 47. Chen W, Liu Y, Courtney HS, Bettenga M, Agrawal CM, Bumgardner JD, et al. In vitro anti-bacterial and biological properties of magnetron co-sputtered silver-containing hydroxyapatite coating. Biomaterials. 2006;27(32):5512-5517.

48. Kassaee MZ, Akhavan A, Sheikh N, Sodaga A. Antibacterial Effects of a New Dental Acrylic Resin Containing Silver Nanoparticles. Journal of Applied Polymeric Science. 2008;110:1699-1703.

49. Dianat O, Ataie M. Gutta-percha coated with nanosilver particles. Invention registered number: 56019, 2008.

50. Shantiaee Y, Dianat O, Mohammad Khani H, Akbarzadeh Baghban A. Cytotoxicity comparison of nanosilver coated gutta-percha with Guttaflow and normal gutta-percha on L929 fibroblast with MTT assay. Beheshti University Dental Journal. 2011;29:62-68.

51. Monteiro DR, Gorup LF, Takamiya AS, de Camargo ER, Filho AC, Barbosa DB. Silver distribution and release from an antimicrobial denture base resin containing silver colloidal nanoparticles. J Prosthodont. 2012;21(1):7-15.

52. Subba Rao CVS, Vanajasan PP, Chandana VS. Scope of Biomaterials in Conservative Dentistry and Endodontics. Trends in Biomater Artif Organs. 2011;25:75-78. 